\title{
Prediction of Tractive Limitations of a Rigid Wheel on Loose Soil
}

\author{
Kenji Nagaoka ${ }^{1}$, Noriaki Mizukami ${ }^{2}$, and Takashi Kubota ${ }^{3}$ \\ ${ }^{1}$ Department of Aerospace Engineering, Tohoku University,nagaoka@astro.mech.tohoku.ac.jp \\ ${ }^{2}$ Department of Space and Astronautical Science, The Graduate University for Advanced Studies, mizukami@nnl.isas.jaxa.jp \\ ${ }^{3}$ Japan Aerospace Exploration Agency, kubota@isas.jaxa.jp
}

\begin{abstract}
Predicting the tractive limitations of locomotion gears is of critical importance in a robotic vehicle system on natural terrain. In particular, it is imperative for an unmanned mobile robot to avoid getting stuck in loose soil to retain its mobility. From this perspective, this paper attempts to theoretically predict the limitations of a typical wheel in such loose soil based on traditional soil-wheel interaction models. In the case that the model brings a negative drawbar pull exerted by the wheel under any kinematic slip condition, we analytically investigated the limitations using a ratio of sinkage over the wheel radius. Predicted limitations are suggested for design optimization and control of the wheeled vehicle. Furthermore, this paper presents the relationship between the model analysis and a practical single wheel test, which provides significant guidelines for usage of the experimental results.
\end{abstract}

\section{Keywords}

rigid wheel, tractive limitations, loose soil, soil-wheel interaction, prediction model

\section{INTRODUCTION}

In human history, the construction of a wheel is one of the most outstanding achievements in engineering. Although the exact origin of the wheel is unknown, according to one theory, it is believed that a first wheeled chariot was invented by Sumerian more than 5000 years ago. A wheel is generally regarded as a simple and efficient locomotion gear. It has thus been applied to various means of transportation on rough terrains as well as on paved roads because of its high capability for locomotion. However, while the wheel has broad utility, it has a potential disadvantage on locomotion over deformable ground such as soil, mud or marsh. In particular, wheeled vehicles often get stuck in loose soil, a situation that is quite hard to avoid. Recently, NASA's Mars Exploration Rover Spirit was stuck in loose sand on Mars. Despite repeated attempts to free the rover, NASA ultimately gave up freeing it from the sand trap [NASA Mission News, 2010]. This instance motivates a discussion on tractive limitations of the wheel for traveling over loose soil.

On the other hand, an academic discipline addressing vehicle-terrain interactive mechanics has attracted attention since the mid-20th century. This discipline is called terramechanics, and it has played a considerable role in the study of off-road vehicles on difficult terrains. Accumulated findings in terramechanics can provide guidelines when discussing traveling perform- ance of a wheel on soil. One of the mainstream approaches in terramechanics is research on semi-empirical models with regard to steady states of the wheels on the soil [Bekker, 1956; Reece, 1965; Bekker, 1969; Wong, 2001; Muro and O'Brien, 2004]. The modeling approach has also contributed to analyze the wheel's traveling performance in extreme environments such as lunar and planetary surfaces. In addition, the terramechanics model has been currently expanded to soil parameter estimation from a geological standpoint [Iagnemma and Dubowsky, 2005; Ray, 2009]. However, the applicable state of the models is limited to a steady state with constant slippage and rotation torque of the wheel, and therefore, the models cannot be easily applied to simulations on vehicle dynamics. One possible analysis on wheel locomotion mechanics based on the models is to examine only the steadystate limitations of wheel traction. Nevertheless, the limitations of the wheel traction on loose soil have not explicitly been elaborated. The limitations represent an unmovable state with plenty of slippage. As mentioned previously, a mobile robot should not stuck in soil. Hence, an investigation of the limitations is a challenging subject and is useful for providing a clue as to how a robot can prevent getting stuck in soil. This paper indicates the limitations by using the terramechanics models in an attempt to answer the question, "What parameter is a key factor to avoid getting stuck in loose soil?" In particular, the key factor is quantitatively examined through simulation analyses. Furthermore, the application methodology of the models is described with conventional experimental 
approaches.

\section{SOIL-WHEEL INTERACTION MODEL}

It is empirically found that getting stuck in soil is attributed to the collapse of wheel rotation on the soil surface. Until now, terramechanics has contributed to formulate the soil-wheel interaction by applying some empirical parameters. As fundamental models developed in terramechanics, the equilibrium of a wheel, moving on horizontal ground with constant velocity, can be expressed as follows [Wong, 2001].

$D P=r b \int_{\theta}^{\theta_{f}}(\tau \cos \theta-\sigma \sin \theta) d t$,

$F_{Z}=r b \int_{\theta_{r}}^{\theta_{f}}(\tau \sin \theta-\sigma \cos \theta) d t$

where $r$ is the wheel radius, $b$ is the wheel width, $\theta$ is the wheel angle, $\theta_{f}$ is the entry angle, and $\theta_{r}$ is the exit angle. Further, $\sigma$ is the normal stress beneath the wheel and $\tau$ is the shear stress in the longitudinal direction of the wheel (see Figure 1). By these equations, the contact forces (the drawbar pull DP and the vertical force $F_{Z}$ ) can be analytically calculated from the steady motion of the wheel. The stresses $\sigma$ and $\tau$ are evaluated as average values across the wheel width, and the wheel is assumed to not have any lugs, grousers or treads on its surface. For evaluating the wheeled tractive performance, we introduce the wheel slip in the direction of wheel travel. Slip is a principal parameter used in an elicitation process of the above equations. In this paper, the wheel slip s is defined as a function of the wheel's translational and circumferential velocities as follows [Bekker, 1956].

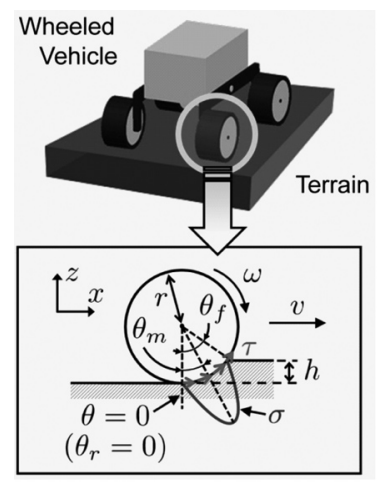

Fig. 1 Soil-wheel interaction model

$s=\left\{\begin{array}{l}1-\frac{v}{r \omega}: 0 \leq s \leq 1: \\ \frac{r \omega}{v}-1:-1 \leq s \leq 0\end{array}\right.$,

where $v$ is the wheel's translational velocity and $\omega$ is the wheel's angular velocity. The wheel's circumferential velocity is represented by $r \omega$. The wheel states

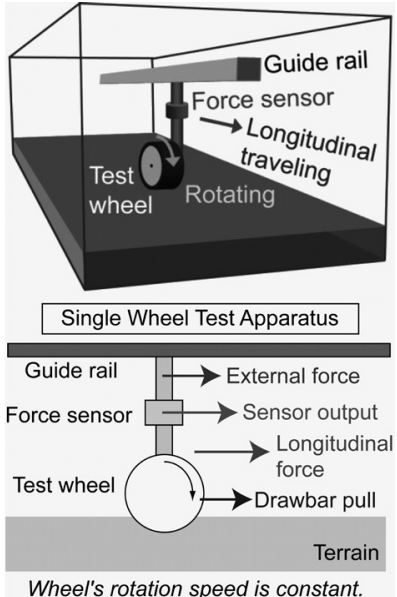

Fig. 2 Schematic of single wheel test system

are typically divided into three states: self-propelled, tractive driving and braking. Practically, in both the self-propelled and the tractive driving states, Eq. (3) results in $0 \leq s \leq 1$, and in the braking state, it results in $-1 \leq s \leq 0$. In this paper, wheel motion under $0 \leq s$ $\leq 1$ is elaborated. For experimentally implementing the slip range $0 \leq s \leq 1$, we can use the single wheel test system shown in Figure 2. A detailed description of the test system is described in the subsequent section. Referring to the soil-wheel interaction shown in Figure 1, $\theta$ is defined as a positive value in a counterclockwise rotation from the $\theta=0$ line (Figure 1). Let $\theta_{f}$ be geometrically written with the wheel sinkage $h$ as the following equation.

$\theta_{f}=\cos ^{-1}\left(1-\frac{h}{r}\right)$

Figure 3 plots the relationship between $\theta_{f}$ and $h / r$, where $h / r$ is defined as the sinkage ratio. According to Figure 3, for instance, $h / r$ becomes approximately 0.29 at $\theta=45 \mathrm{deg}$. The anglular components of $\sigma$ and $\tau, \sin \theta$. and $\cos \theta$, respectively, are affected by the increase in $h / r$.

With respect to $\sigma$, a well-known formulation is available as follows [Bekker, 1956; Wong, 2001].

$\sigma=\left\{\begin{array}{l}\text { if } \theta_{m} \leq \theta \leq \theta_{f} \\ \left(\frac{k_{c}}{b}+k_{\varnothing}\right) r^{n}\left(\cos \theta-\cos \theta_{f}\right)^{n} \\ \text { otherwise } \\ \left(\frac{k_{c}}{b}+k_{\varnothing}\right) r^{n}\left\{\begin{array}{c}\cos \left[\theta_{f}-\frac{\left(\theta_{f}-\theta_{m}\right)}{\theta_{m}} \theta\right. \\ \left.\times \theta_{m}\right]-\cos \theta_{f}\end{array}\right\}^{n},\end{array}\right.$

where $n$ is the pressure sinkage ratio, $k_{c}$ and $k_{\varnothing}$ are the deformation modulus for the cohesion stress $C$ and the internal friction angle $\varnothing$, respectively. Here, $\theta_{r}$ is sim- 


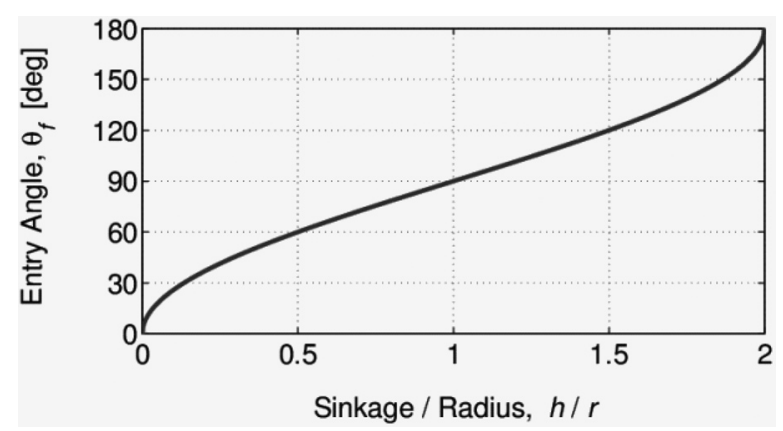

Fig. 3 Relationship between $\theta_{f}$ and $h=r$

ply assumed zero, as shown in Figure 1 [Wong, 2001]. Furthermore, to determine the distribution profile of $\sigma$, the maximum stress angle $\theta_{m}$ can be represented as follows [Onafeko and Reece, 1967].

$\theta_{m}=\left(c_{1}+c_{2} s\right) \theta_{f}$

where $c_{1}$ and $c_{2}$ are the stress coefficients depending on the soil-wheel interaction. In Eq. (6), $c_{1}=0.4$ and 0 $\leq c_{1} \leq 0.3$ are generally suggested by the empiricallybased results [Wong, 2001; Iagnemma and Dubowsky, 2005]. Most terramechanics studies assume that $\tau$ acts along the wheel surface. Based on past research [Janosi and Hanamoto, 1961], $\tau$ of the loose soil can be empirically obtained as follows.

$\tau=(C+\sigma \tan \varnothing)\left[1-\exp \left(-\frac{j}{K}\right)\right]$,

where $j$ is the soil displacement and $K$ is the soil deformation modulus. The shear strength $(\mathrm{C}+\sigma \tan \varnothing)$ is also defined as $\tau_{\max }$. Given the slip velocity, $r \omega-v$ $\cos \theta, j$ can be determined by the following equation [Wong, 2001].

$j=r\left[\theta_{f}-\theta-(1-s)\left(\sin \theta-\sin \theta_{f}\right)\right]$,

\section{PARAMETRIC ANALYSIS \\ 3.1 Fundamental simulation conditions}

The wheel models can be outlined by the single wheel test shown in Figure 2. The test wheel's traveling direction is fundamentally constrained in a forward direction along the guide rail. Furthermore, the simulated soil is set to be dry sand. The parameters of the dry sand are listed in Table 1. Likewise, the nominal parameters of the wheel geometry are set to be $r=0.1$ $\mathrm{m}$ and $b=0.1 \mathrm{~m}$, respectively.

As mentioned previously, the models can be used for calculating $D P$ under steady motion with a constant slip. Thus, we here define $D P=0$ within $0 \leq s \leq 1$ as the tractive limitations. Parameter dependence on $D P$ is first analyzed to elucidate the characteristics of the limitations. In addition, from the empirical results [Yamakawa et al., 2008], $h$ with a slip sinkage effect can be considered as

$h=h_{0}+c_{3} s$,

where $h_{0}$ is the nominal sinkage at $s=0$ and $c_{3}$ is a positive coefficient pertaining to slip. Equation (9) allows one to simulate a tendency that $h$ increases with $s$. Remarks analogous to such a tendency have been experimentally reported in the literature [Jide et al., 1991; Lyasko, 2010]. Thus, the subsequent analyses use two sinkage models, constant $\left(c_{3}=0\right)$ and variable $\left(c_{3} \neq 0\right)$.

\subsection{Simulation analysis}

\subsubsection{Effects of slip and sinkage ratio on stress distri- butions}

The first simulation analysis is of the stress distributions ( $\sigma$ and $\tau$ ), with the dependence on $s$ and $h / r$. As examples of the simulations, the computed stresses under $s=0.2,0.8$ and $h / r=0.2,0.8$ are shown in Figure 4 . These results suggest that $\theta_{m}$ is proportional to $s$ as expressed in Eq. (6). However, the stress distribu-

Table 1 Nominal parameters of dry sand [Wong, 2001; Iagnemma and Dubowsky, 2005]

\begin{tabular}{|l|c|c|c|}
\hline Soil Parameter & Symbol & Value & Unit \\
\hline Internal friction angle & $\varnothing$ & 28 & $\mathrm{deg}$ \\
\hline Cohesion stress & $c_{1}$ & 1000 & $\mathrm{~Pa}$ \\
\hline Pressure-sinkage modulus for internal friction angle & $k_{c}$ & 1523.4 & $\mathrm{kN} / \mathrm{m}^{n+2}$ \\
\hline Pressure-sinkage modulus for cohesion stress & $k_{\varnothing}$ & 900 & $\mathrm{~N} / \mathrm{m}^{n+1}$ \\
\hline Deformation modulus & $K$ & 0.025 & $\mathrm{~m}$ \\
\hline Pressure-sinkage ratio & $n$ & 1.1 & - \\
\hline Coefficient of normal stress distribution & $c_{1}$ & 0.4 & - \\
\hline Coefficient of normal stress distribution & $c_{2}$ & 0.15 & - \\
\hline
\end{tabular}



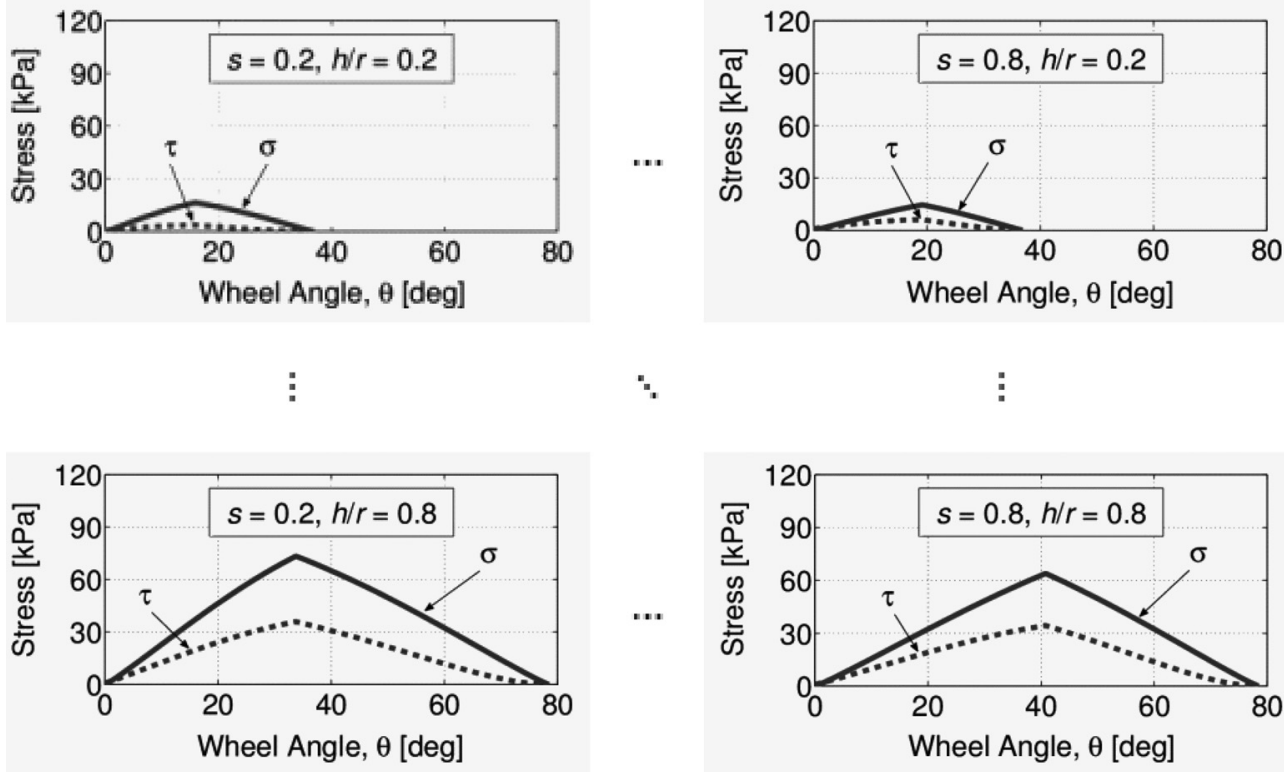

Fig. 4 Soil stress distributions along $\theta$

tions are totally affected by $h / r$ and not by $s$. From a quantitative perspective, the maximum values of the stresses become more than quadrupled with the increase of $h / r$ from 0.2 to 0.8 .

\subsubsection{Effects of slip and wheel geometry on shear} function

The second simulation analysis demonstrates the characteristics of the shear function in Eq. (7). The shear

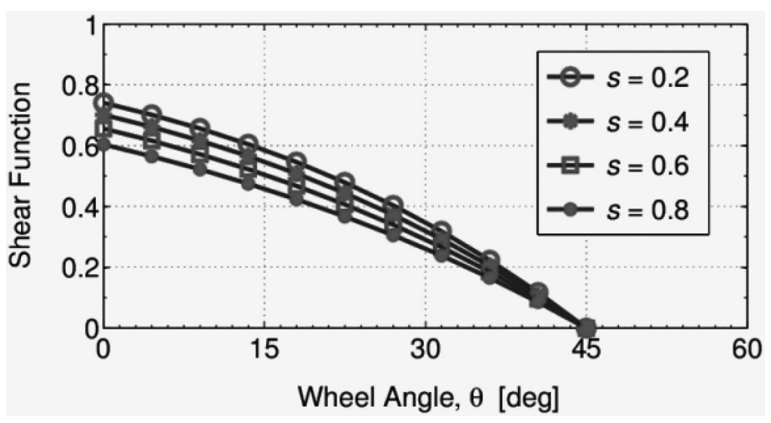

(a) Kinematic effect of $s$ with $r / K=1$

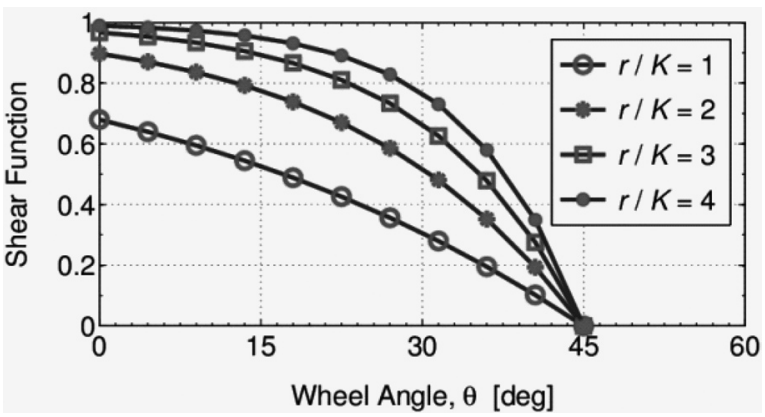

(b) Geometric effect of $r / K$ with $s=0.5$

Fig. 5 Shear function $\tau=\tau_{\max }$ along $\theta$ (where $r$ and $h$ are constant) function, which can be also represented as $\tau=\tau_{\max }$, is an important index for exerting positive $D P$. Figure 5 plots the effects of $s$ and $r / K$ on the shear function with a constant $\theta_{f}$. From these results, one can find that $r / K$ is more effective than $s$ in the change of the shear function. According to Eq. (7), the increase in $r /$ $K$ allows a reduction in the shear function exponentially. Thus, larger $r$ should be designed to obtain the appropriate shear stress because $K$ is basically one of the uncontrollable soil parameters. On the other hand, it is concluded that $\tau$ does not depend much on $s$ itself.

\subsubsection{Effects of sinkage ratio on drawbar pull and vertical force}

Through the above analyses, it was found that $h /$ $r$ is the significant parameter for wheel locomotion. Hence, we next investigate the dependence of $h / r$ on $D P$ and $F_{Z}$, which are fundamental indexes to indicate the tractive performance. Figure 6 shows the simulation results. While changing $s$ results in a certain amount of change in $D P$ and $F_{Z}, h / r$ is obviously a dominant parameter. The main reason of this result is because the magnitudes of the sine and the cosine components are reversed over $\theta=45 \mathrm{deg}$. The functional components affect $\sigma$ and $\tau$ for $D P$; therefore, these are subject to $h / r$ as shown by the relationship in Figure 3. Consequently, the semi-empirical model shows that the $s-D P$ curve shifts downward with the increase in $h$, as shown in Figure 7.

\subsubsection{Effects of slip sinkage on drawbar pull}

As the next step, the characteristics of $D P$ with the practical sinking behavior are discussed. Figure 8 shows the relationship between $D P$ and $s$ with the 


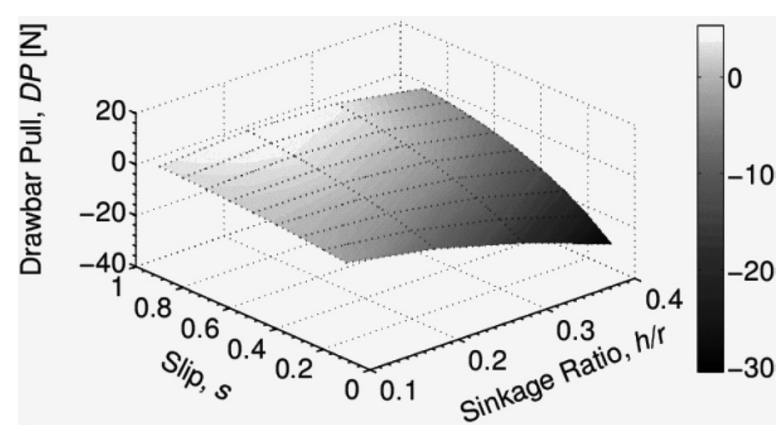

(a) $D P$

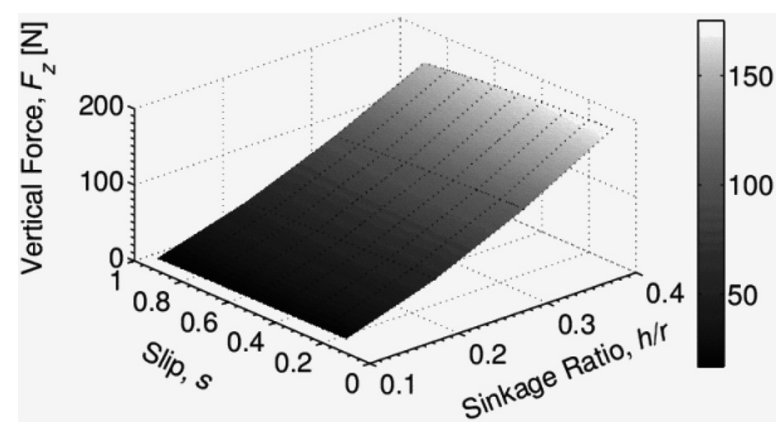

(b) $F_{Z}$

Fig. 6 Three-dimensional distributions of contact forces versus $s$ and $h / r$

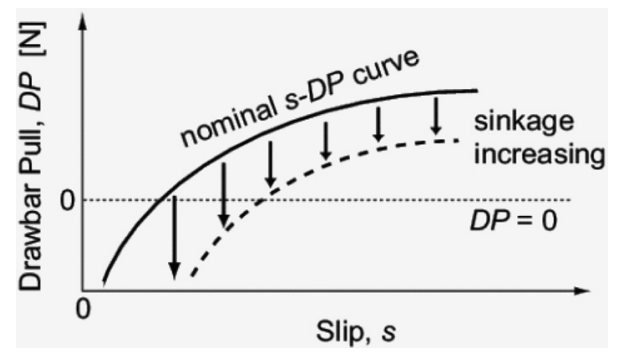

Fig. $7 s-D P$ curve shifting with increase in $h$

slip sinkage effect: $h_{0}=0.005 \mathrm{~m}$ and $c_{3}=0.015,0.03$, 0.045 . The simulated $D P$ bears a proportionate relationship to $s$ at lower $c_{3}$ (e.g., $c_{3}=0.015$ ). On the other hand, it is found that $D P$ consistently becomes less than zero with larger $c_{3}$ (e.g., $\left.c_{3}=0.045\right)$. This case is exactly within the tractive limitations. Therefore, we

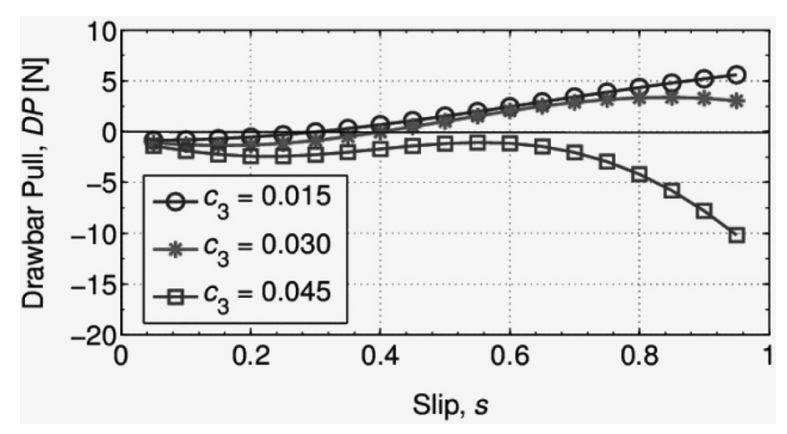

Fig. $8 s-D P$ curve with slip sinkage effect can conclude that $h / r$ is the key factor for preventing a wheel from getting stuck in soil.

\section{TRACTIVE LIMITATIONS \\ 4.1 Simulated tractive limitations}

In this section, we present the tractive limitations of the wheel by a function of the sinkage ratio $h / r$. Figure 9 plots the minimal values of $h / r$ so that $D P$ is less than zero with variable $r$, in which dry sand was targeted. From the resulting curves at lower $r$ in Figure 9 (a), it can be seen that the minimal $h / r$ basically increases with the increase in $r$. This implies that the large wheel radius delivers a better performance on the soil. However, this advantage is saturated beyond a certain value of $r$, where the minimal $h / r$ reaches a steady value over $r=0.5 \mathrm{~m}$. With the normalization of $r$ by $K$, the fundamental characteristics of the minimal $h / r$ become marked as shown in Figure 9 (b).

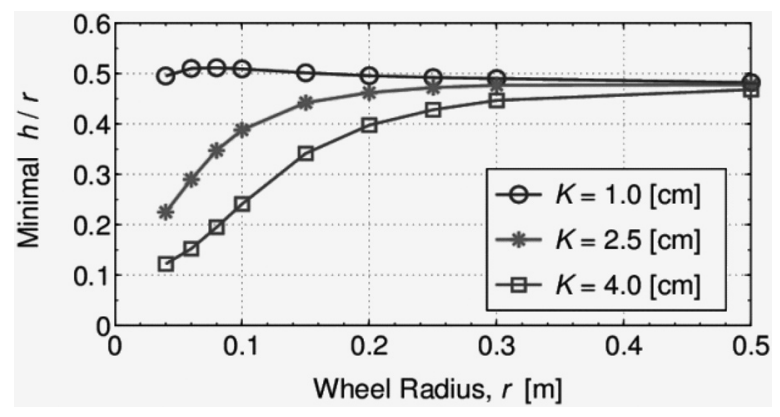

(a) Effect of $K$

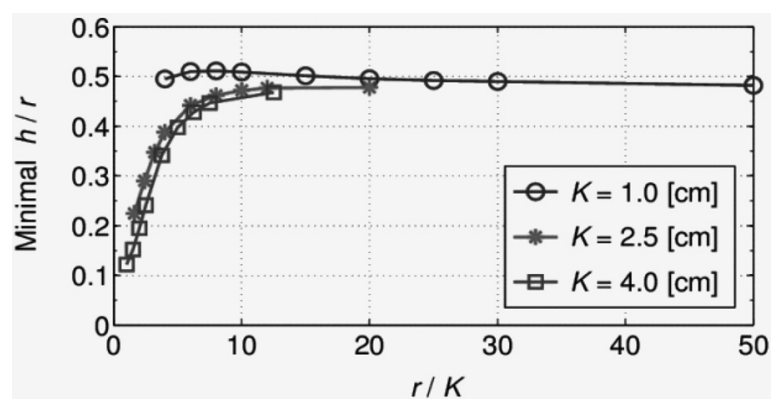

(b) Normalized curves

Fig. 9 Tractive limitations of the wheel with respect to variable $r$ : dry sand

Next, we ran additional simulations on lunar fine soil. The objective of these simulations is to investigate the effects of variable soil parameters. According to a previous report [Heiken et al., 1991], lunar soil has a wide grain size distribution and its relative density varies greatly. Thus, its physical parameters vary depending on its density. Moreover, the soil parameters to be used in the models can be determined by past data from lunar soil tests [Wallace and Rao, 1993; Heiken et al., 1991]. This paper focuses on the dependence of $\varnothing$ and $C$ with the tractive limitations. 
Table 2 Nominal parameters of lunar soil [Wallace and Rao, 1993; Iagnemma and Dubowsky, 2005]

\begin{tabular}{|l|c|c|c|}
\hline Soil Parameter & Symbol & Value & Unit \\
\hline Internal friction angle & $\varnothing$ & 35 & $\mathrm{deg}$ \\
\hline Cohesion stress & $c_{1}$ & 170 & $\mathrm{~Pa}$ \\
\hline Pressure-sinkage modulus for internal friction angle & $k_{c}$ & 814.4 & $\mathrm{kN} / \mathrm{m}^{n+2}$ \\
\hline Pressure-sinkage modulus for cohesion stress & $k_{\varnothing}$ & 1379 & $\mathrm{~N} / \mathrm{m}^{n+1}$ \\
\hline Deformation modulus & $K$ & 0.0178 & $\mathrm{~m}$ \\
\hline Pressure-sinkage ratio & $n$ & 1.0 & - \\
\hline Coefficient of normal stress distribution & $c_{1}$ & 0.4 & - \\
\hline Coefficient of normal stress distribution & $c_{2}$ & 0.15 & - \\
\hline
\end{tabular}

The nominal parameters of the lunar soil are shown in Table 2. Figure 10 plots the simulation results with the lunar soil. As a result, we can suggest that. greatly affects the limitations, as shown in Figure 10 (b). Although the change in $C$ is not discussed here, we indeed confirmed that the effect of $C$ is much smaller than that in $\varnothing$. In the case of lunar soil, the converging minimal $h / r$ becomes greater than that of dry sand.

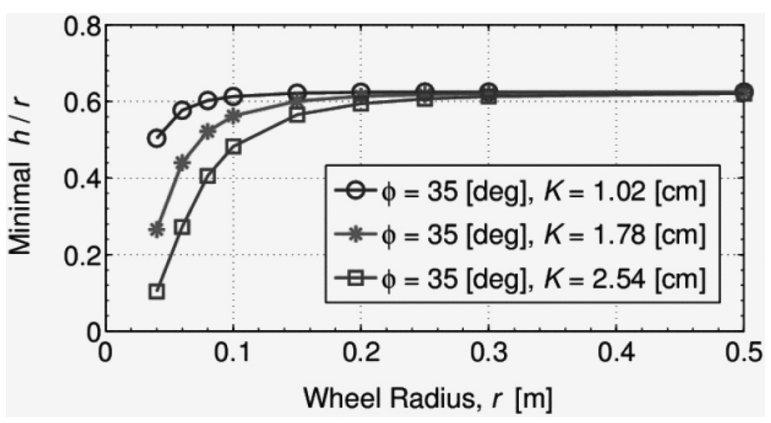

(a)Effect of $K$

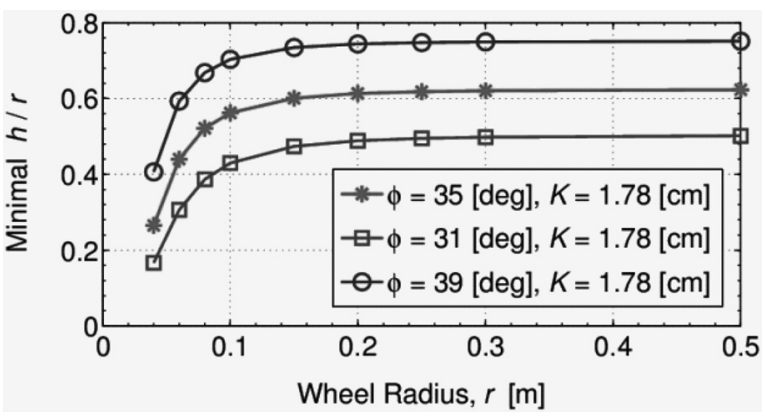

(b)Effect of $\varnothing$

Fig. 10 Tractive limitations of the wheel with respect to variable $r$ : lunar soil

\subsection{Prediction results}

Based on the simulation results of the tractive limitations, we attempted to draw a predictive approximation curve of the minimal $h / r$ with respect to the tractive limitations. Given that the soil-wheel interaction parameters $k_{c}, k_{\varnothing}$, and $n$ are constant, the minimal $h / r$ is mainly governed by $\varnothing$. In general, the wheel radius $r$ can be selected by a designer. Thus, this paper primarily discusses the minimal $h / r$, which is a converging minimal one at $s=1$ (specifically, the minimal $h / r$ at $r=0.5 \mathrm{~m}$ ). On the other hand, $\varnothing$ affects the drawbar pull as a function of $\tan \varnothing$. Thus, we defined the prediction curve as $h / r=k_{p} \tan \varnothing$, where $k_{p}$ is a prediction coefficient. In accordance with Figures. 9 and 10, the prediction curve was finally given by the following equation.

$\frac{h}{r}=0.9 \tan \varnothing$.

Figure 11 shows the prediction curve. This curve confirmed that the resulting prediction better meets the simulation plots of both types of soils (dry sand and lunar soil).

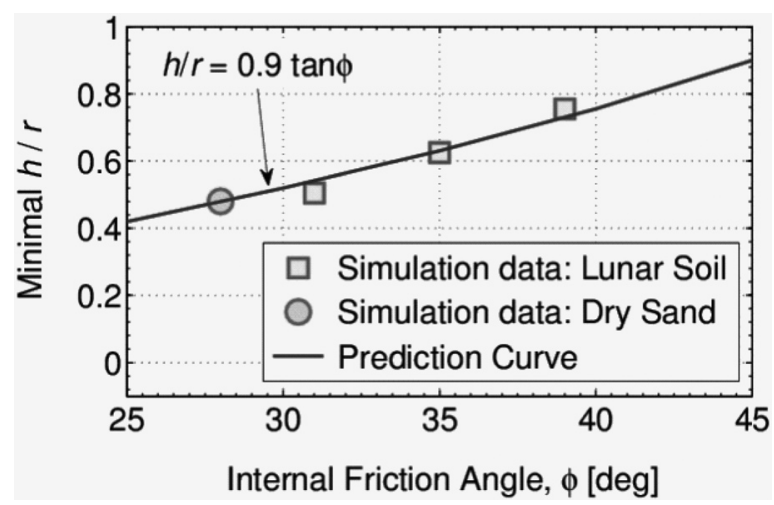

Fig. 11 Prediction curve of the tractive limitations at convergence region

\section{DISCUSSION}

Figure 2 shows the fundamental configuration of the single wheel test apparatus. The wheel is connected to the guide rail with an attached a force sensor. The guide rail can actively shift back and forth with the 


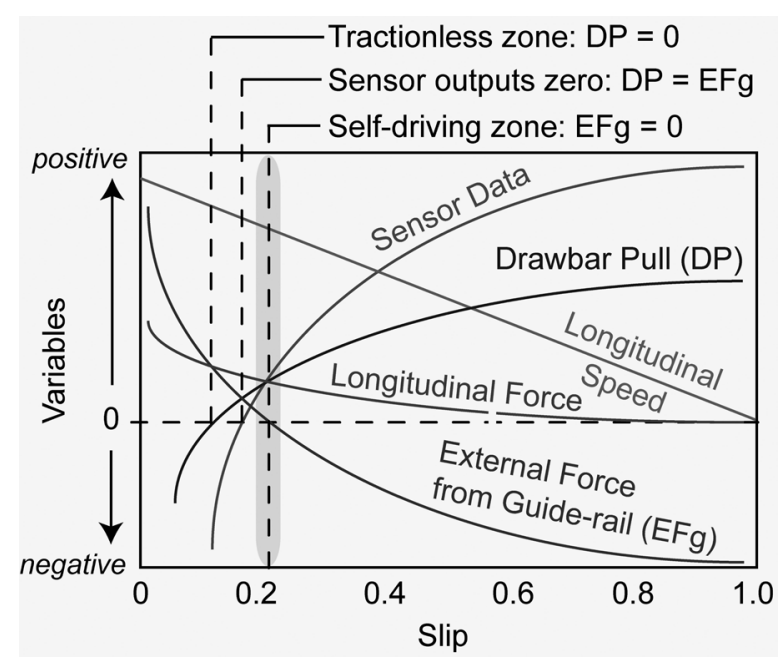

Fig. 12 Tendency resulted by single wheel tests

help of a motor, and it can move freely in a vertical direction without resistance. Accordingly, the total force, governing the wheel motion, can be represented as the summation of the $D P$ and the external force exerted by the guide rail (hereafter, $E F_{g}$ ). Using this apparatus, we can obtain the sensor output, the vertical and horizontal displacements, and $E F_{g}$. Assuming that the wheel travels horizontally under constant $\omega$ and $h$, the correlation of all data will be as shown in Figure 12. This indicates the self-propelled state of the wheel is limited at a certain point in the $s-D P$ curve, where the external force becomes zero. The acquired $D P$ appears with $E F_{g}$ as paired data so that $D P-E F_{g}$ becomes zero under the steady states. On the other hand, a multi-wheel system is a possible solution for wheeled locomotion in practice. Most of the data acquired by the single wheel tests simulate just one wheel of a multi wheel system. Therefore, we must take care to understand that the generation of $D P$ in the $s-D P$ curve is inextricably associated with which the other wheels shall, in total, generate the external force exerted from the guide rail as their drawbar pull.

\section{CONCLUSION}

This paper analytically investigated tractive limitations of a wheel on loose soil by applying semiempirical soil-wheel interaction models. Although the models simulated just steady states, we provided the static limitations of the wheel traction by evaluating negative drawbar pulls under any slippage. In particular, this paper clarified that the sinkage ratio $h / r$ is the most significant factor for preventing the wheel from getting stuck in loose soil. In accordance with the simulation analyses, it was found that the minimal $h$ / $r$ approaching tractive limitations greatly depends on the internal friction angle of the soil. Given the practical parameter range, $h / r$ must be theoretically made less than $0.78\left(\theta_{f}=51.26 \mathrm{deg}\right)$ even if a high frictional soil and a large-diameter wheel were assumed. Consequently, the result of this paper can contribute to optimization of wheel design. Furthermore, the application methodology of the findings obtained from the single wheel tests to a mobile robot equipping multi wheels was discussed. As work in progress, we are planning experimental evaluations for validating the prediction model.

\section{References}

Bekker, M. G., Theory of Land Locomotion, University of Michigan Press, 1956.

Bekker, M. G., Introduction of Terrain-Vehicle Systems, University of Michigan Press, 1969.

Heiken, G. H., D. T. Vaniman, and B. M. French, Lunar Sourcebook: A User's Guide to the Moon, Cambridge University Press, 1991.

Iagnemma, K., and S. Dubowsky, Mobile Robots in Rough Terrain, Springer, 2005.

Janosi, Z. J., and B. Hanamoto, The analytical determination of drawbar pull as a function of slip for tracked vehicle, Proceedings of the 1st International Conference on Terrain-Vehicle Systems, 707736, 1961.

Jide, Z., W. Zhixin, and L. Jude, The way to improve the trafficability of vehicles on sand, Proceedings of the 5th European Conference on Terrain-Vehicle Systems, 121-126, 1991.

Lyasko, M., Slip sinkage effect in soil-vehicle mechanics, Journal of Terramechanics, Vol. 47, No. 1, 21-31, 2010.

Muro, T., and J. O’Brien, Terramechanics, A. A. Balkema Publishers, 2004.

NASA Mission News, http://www.nasa.gov/ mission_ pages/mer/news/mer20100126.html, 2010.

Onafeko, O., and A. R. Reece, Soil stresses and deformations beneath rigid wheels, Journal of Terramechanics, Vol. 4, No. 1, 59-80, 1967.

Ray, L. E., Estimation of terrain forces and parameters for rigid-wheeled vehicles, IEEE Transactions on Robotics, Vol. 25, No. 3, 717-726, 2009.

Reece, A. R., Principles of soil-vehicle mechanics, Proceedings of the Institution of Mechanical Engineers, Vol. 180 (2A), No. 2, 45-66, 1965.

Wallace, B. E., and N. S. Rao, Engineering elements for transportation on the lunar surface, Applied Mechanics Reviews, Vol. 46, No. 6, 301-312, 1993.

Wong, J. Y., Theory of Ground Vehicles (Third Edition), John Wiley \& Sons, 2001.

Yamakawa, J., Yoshimura, O., and Watanabe, K., Development of tire model on dry sand by model size experiment (first report), Transactions of the Society of Automotive Engineers of Japan, Vol. 39, No. 
6, 41-46, 2008.

(Received February 18, 2012; accepted May 8, 2012) 